\title{
LSST Active Optics System Software Architecture
}

\author{
Sandrine J. Thomas ${ }^{\mathrm{a}}$, Paul Lotz ${ }^{\mathrm{a}}$, Srinivasan Chandrasekharan ${ }^{\mathrm{a}}$, Bo Xin ${ }^{\mathrm{a}}$, Charles Claver ${ }^{\mathrm{a}}$, \\ George Angeli ${ }^{\mathrm{a}}$, Jacques Sebag ${ }^{\mathrm{a}}$, and Gregory P. Dubois-Felsmann ${ }^{\mathrm{b}}$ \\ ${ }^{a}$ LSST, 950 N. Cherry Av, Tucson, AZ, USA \\ ${ }^{\mathrm{b}}$ Caltech, 1200 E California Blvd, Pasadena, CA 91125, USA
}

\begin{abstract}
The Large Synoptic Survey Telescope (LSST) is an 8-meter class wide-field telescope now under construction on Cerro Pachón, near La Serena, Chile. This ground-based telescope is designed to conduct a decade-long time domain survey of the optical sky. In order to achieve the LSST scientific goals, the telescope requires delivering seeing limited image quality over the 3.5 degree field of view. Like many telescopes, LSST will use an Active Optics System (AOS) to correct in near real-time the system aberrations primarily introduced by gravity and temperature gradients. The LSST AOS uses a combination of 4 curvature wavefront sensors (CWS) located on the outside of the LSST field-of-view. The information coming from the 4 CWS is combined to calculate the appropriate corrections to be sent to the 3 different mirrors composing LSST. The AOS software incorporates a wavefront sensor estimation pipeline (WEP) and an active optics control system (AOCS). The WEP estimates the wavefront residual error from the CWS images. The AOCS determines the correction to be sent to the different degrees of freedom every 30 seconds. In this paper, we describe the design and implementation of the AOS. More particularly, we will focus on the software architecture as well as the AOS interactions with the various subsystems within LSST.
\end{abstract}

Keywords: Large Synoptic Survey Telescope, Active Optics, Wide Field of View, Curvature Sensing

\section{INTRODUCTION}

The Large Synoptic Survey Telescope (LSST) is an 8.4 meter diameter telescope now under construction on Cerro Pachon in Chile. ${ }^{1,2}$ The telescope is composed of three aspheric mirrors: the $8.4 \mathrm{~m}$ primary mirror (M1), a $3.4 \mathrm{~m}$ secondary mirror (M2) and a 5m tertiary mirror (M3). The primary and tertiary mirrors form a single monolithic mirror called the M1M3 $3^{3,4}$. LSST has one instrument, a three-lens camera that directs the light path onto a 3.2 gigapixel camera, with a field-of-view (FOV) of 3.5 degrees. ${ }^{5}$ The camera has 6 different filters, $\mathrm{u}, \mathrm{g}, \mathrm{r}, \mathrm{i}, \mathrm{z}, \mathrm{y}$. The overall system image quality budget for the LSST is $0.4 \operatorname{arcsec}$ FWHM with 0.25 arcsec allocated to the telescope and 0.3 arcsec associated to the camera. LSST is a seeing-limited telescope and thus these performance do not include the errors coming from the atmosphere.

In order to optimize the image quality across the 3.5 degree FOV of the camera, LSST relies on an Active Optics System (AOS). Both M1M3 and M2 are equipped with actuators that allow for the AOS to control the shape of the mirror surface and their position. ${ }^{6}$ In addition, M2 and the camera are on hexapods allowing further positioning adjustment. ${ }^{7}$

The wavefront control is performed by determining a base set of control parameters based on both finite element analysis (FEA) and on-sky open-loop measurements as well as by adding low temporal frequency closed loop corrections determined from real-time wavefront measurements. The open-loop model may take the form of equations or tables. In this paper, we also refer to this as Look-up Table (LUT). The LUT provides near optimum values for all actuator forces and hexapods positions and depends primarily on elevation angle and temperature. Although constructed using FEA models, it will be verified extensively during the commissioning phase.

Further author information: (Send correspondence to S.J.T.)

S.J.T.: E-mail: sthomas@lsst.org, Telephone: 15203188227

Ground-based and Airborne Telescopes VI, edited by Helen J. Hall, Roberto Gilmozzi, Heather K. Marshall, Proc. of SPIE Vol. 9906, 99063B · C 2016 SPIE · CCC code: 0277-786X/16/\$18 · doi: 10.1117/12.2231798 
The corrections given by the LUT, however, do not allow the system to meet the LSST image quality requirements due to non-repeatable and/or unpredictable effects. These effects include temperature errors, wind loads and hysteresis. Accordingly, LSST plans to use real-time wavefront sensor measurements to compensate for these errors to achieve the LSST required image quality. The wavefront sensor selected is a curvature sensor, since the wide-field and the fast beam make the use of the more popular Shack-Hartmann wavefront sensor problematic. Moreover, the curvature sensors can be used in the focal plane, while the Shack-Hartmann is used in the pupil plane. The curvature sensor option avoids non-common path errors. More details on the origin and impact of different environmental perturbations on the LSST image quality are described in a previous paper. ${ }^{8}$

In addition to on-sky wavefront correction, the active optics will be used to conduct alignment verification in coordination with a laser tracker at the beginning of every night or during the night if needed..$^{3,6}$

This paper describes the software architecture of the active optics system. Section 2 presents the different use-cases of the AOS. Section 3 describes the technical aspect of the wavefront sensor both mechanically and relative to software. Section 4 and section 5 detail the steps and functions of the components of the AOS.

\section{OPERATING MODES}

\subsection{Normal Operation and Engineering Operation}

Normal operation is defined by routine nighttime observations for which there is no human intervention. The AOS input is 4 individual pairs of intra- and extra-focal images corresponding to the 4 different wavefront sensors. The images come directly from the Data Acquisition system (DAQ) in the Camera Subsystem. In order to maintain the required image quality, the AOS output consists of the bending modes sent to the mirror support system of M1M3 and M2 to control the mirror shapes, and positions sent to the M2 and camera hexapods. The observatory cadence of the LSST telescope is relatively fast since the telescope pointing changes every 39 seconds. These 39 seconds are decomposed into two $16 \mathrm{~s}$ visits (1s of which is to allow the shutter to open and close), a $2 \mathrm{~s}$ readout and a $5 \mathrm{~s}$ slew time. The current baseline plan uses the data from the first visit only and performs the calculation during the second visit.

During normal operation, we will also have an engineering or manual mode. This mode will be used in open loop, meaning that after an image is acquired, the AOS pipeline does not automatically run through and send the corrections to the mirrors, but allows interactions between the AOS and the user. This submode will be used during commissioning or for corrective maintenance during operation. For instance, when the AOS will be operated manually, it will enable local hardware control with a personal computer via the facility control network. Visualization of images and parameters will also be available.

\subsection{Full Focal Plane Array Wavefront Sensing}

The Full Focal Plane Array (FFPA) mode will be used to build and optimize the LUT during commissioning and on occasion during the day as part of calibration. The science focal plane array (FPA) consists of 189 science detectors. ${ }^{5}$ In this FFPA mode, the AOS obtains the data from the Data Management subsystem (DM) due to the required temporal frequency of correction being dramatically reduced. The images originate from the 189 science detectors for each of the different sets of defocus, mostly $\pm 2 \mathrm{~mm}$, as well as WFS images with the camera in focus for reference. At this reference position, the AOS will collect images from both WFS detectors and science detectors. Using the full focal plane for wavefront sensing is useful to relate the wavefront sensor data to image quality in the field.

This mode will also be used during early commissioning using the commissioning camera (ComCam). ComCam has 1 raft with 9 science detectors, therefore leading to a FOV of 0.6 degrees. This mode will help the commissioning team exercise the AOS algorithm and accomplish the first alignment procedure checks. With regards to software and algorithms, this operating mode is a slight divergence from the nominal automatic operation mode, mostly consisting of an increase of number of sensors read and order of operations. 


\subsection{Alignment procedure}

As mentioned earlier, the AOS will also be used at the beginning of each night as an alignment tool to verify and adjust the reference position relative to the LUT, if needed. In other words, at the beginning of each night, the observatory operator aligns the main optical elements (mirrors and camera) using the following tools: ${ }^{6}$

- A look-up-table incorporates the state of the system measured during the previous night. The look-up-table is the set of absolute hexapod positions and mirror shapes in order to achieve the required image quality. Its requirement is defined below. The reference positions are fixed for at least one night.

- A laser tracker works with the beam reflectors to adjust tip/tilt/rotation and translation. More information on the laser tracker is in Araujo et al. ${ }^{3}$

- The curvature wavefront sensor further minimizes the wavefront error. Before the night's observing, the appropriate active optics corrections are applied to the mirror shapes, and the camera and M2 mirror are positioned relative to the M1M3 mirror by their hexapods. The position of the camera and M2 will likely be verified by the laser tracker system. This ensures that the wavefront errors are within the dynamic range of the active optics system.

\section{LSST WAVEFRONT SENSING TECHNICAL DESCRIPTION}

This section gives a more detailed description of the wavefront sensing for LSST. The first subsection presents the optical layout of the camera dedicated to wavefront sensing and the second section focuses on the software general architecture.

As mentioned in the introduction, the optical wavefront sensor chosen for LSST is a curvature wavefront sensor, leading to the need to have sets of two images with a defined focus offset between them. To satisfy an additional constraint to reduce or eliminate non-common path errors, the wavefront sensor is located in the focal plane of the camera along with the other science sensors.

The wavefront sensor is composed of four sensors manufactured by the Imaging Technology Laboratory (ITL) at the University of Arizona. The sensors are located at the corner of the focal plane as shown on Figure 1-left. Each wavefront sensor is composed of two defocused detectors of $2 \mathrm{k} \times 4 \mathrm{k}$ pixels as shown on Figure 1-right. The optimal defocus was the result of a trade-off study, including impact of signal-to-noise, atmospheric turbulence, non-linearity of the wavefront sensor and sky coverage. ${ }^{9}$

The FOV area of each wavefront sensing detector is 6.8 arcminutes $\times 13.7$ arcminutes. Figure 1-right shows a simulation of the defocused images on the full FOV of one of the four wavefront sensors, using a photon simulation tool called PhoSim. ${ }^{10}$ These simulations are executed using a bright star catalog such as the catalog from the United State Naval Observatory (USNO) led by Monet et al. ${ }^{11}$ or internal catalogs. ${ }^{12}$

The images in Figure 1-right demonstrate some of the difficulties in using the LSST wavefront sensor images to reconstruct the wavefront and calculate the best set of corrections to apply to the different degrees of freedom (presented in section 5). The images show blending issues where two stars are so close to another that the two donuts overlap, resulting in difficulties in the estimation of the wavefront. Some of the donuts also have a low signal-to-noise ratio. The two images show the differences in star counts that can occur even for very close fields. The Active Optics System (AOS) is responsible for measuring the residual wavefront error from the defocused images and estimating the correct bending modes to send to the mirror control systems (M1M3 and M2) and the positions to send to the hexapods (M2 and camera). The AOS is part of the Telescope Control System. ${ }^{13}$

Figure 2 describes the inputs and the output to the AOS or interfaces. The interfaces to the AOS are of several types: interfaces to other subsystems (DM and Camera), and interfaces to the different controllers (M1M3, M2 and the hexapods). The AOS exchanges messages with other systems using a publish-subscribe middleware solution based on an implementation of the Data Distribution Service specification. ${ }^{14}$

Input interfaces. In normal operation the AOS requires crosstalk corrected images from the Camera Data Acquisition System; these images arrive via a custom push data interface. Still in normal operation, the AOS will need to know the state of the camera (selected filter, for example); this information comes from the Observatory Control System (OCS). ${ }^{15}$ Finally, the baseline requires the use of calibration images and data such as 

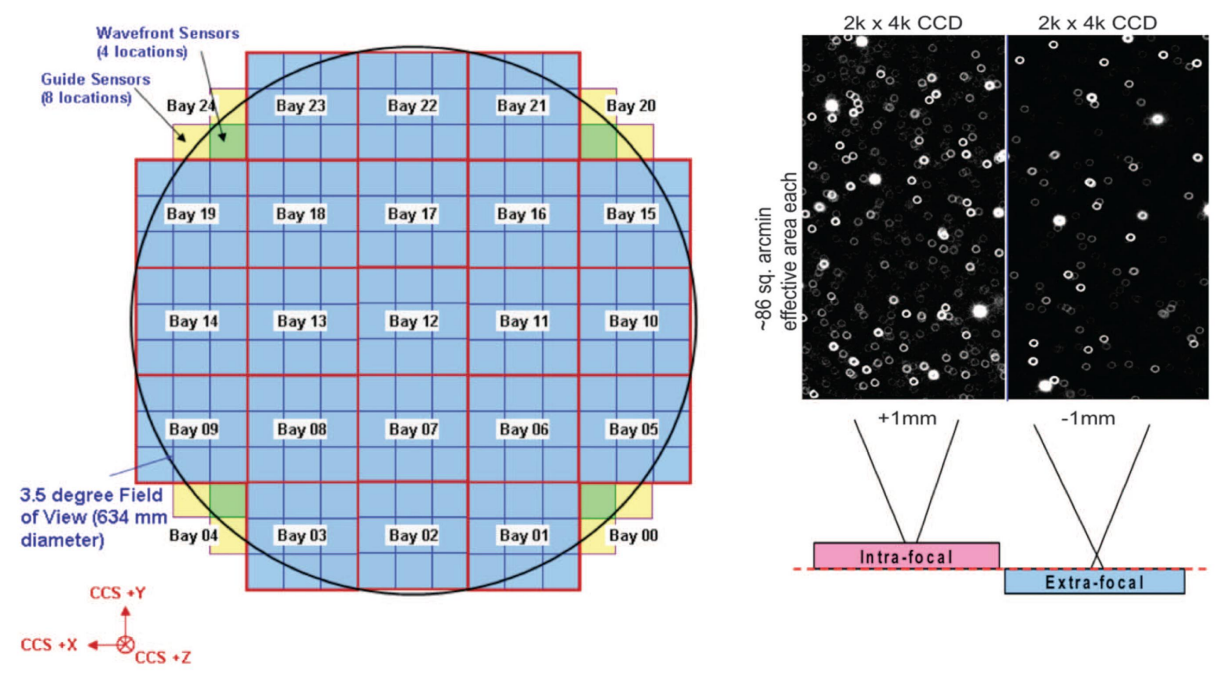

Figure 1. Focal plane layout of the detector. The left drawing shows the full focal plane with the science sensors, the wavefront sensors and the guide sensors. The wavefront sensors and the guide sensors will have vignetting effects as well as distortion that must be taken into account in the wavefront estimation. The right panel shows simulated intra and extra images for one of the wavefront sensors. They were obtained using a complex simulation tool called PhoSim. ${ }^{10}$

flat field images or bad pixels maps as described in section 4.1. This metadata comes from the Data Management subsystem, once a night.

Output interfaces. The AOS outputs bending modes and position information to the different controllers listed in section 5 .

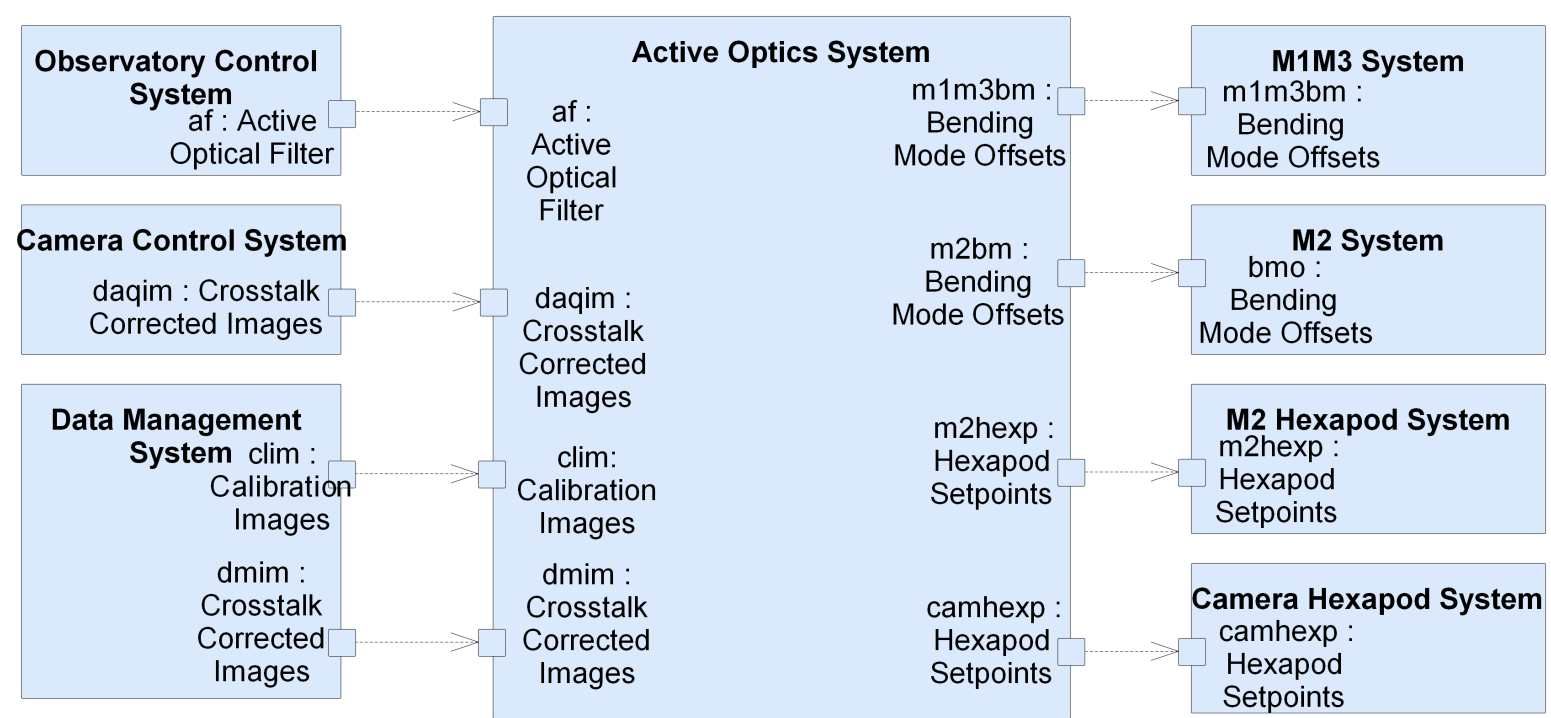

Figure 2. AOS flow diagram showing inputs and outputs between different components. The AOS receives the images either from the camera or the DM subsystems. It also receives telemetry from the Observatory Control System. The output to the AOS is sent to the different mirrors and hexapods controllers. The information sent is the bending modes offsets for the mirrors and the positions for the hexapods/rotator.

The AOS includes the Wavefront Estimation Pipeline (WEP) and the Active Optics Control System (AOCS). 
Each entity will be described in the following section. The WEP sends to the AOCS a set of Zernike coefficients describing the wavefront error for each sensor -4 in normal operation mode and 189 in FFPA mode.

\section{WAVEFRONT ESTIMATION PIPELINE}

As mentioned in the introduction, the Wavefront Estimation Pipeline (WEP) is the algorithm responsible for estimating the wavefront errors in annular Zernike coefficients associated with each of the corner rafts. In normal operation (see section 2), the inputs to the WEP are an intra-focal image and an extra-focal image coming from each of the four corner rafts.

The WFS data are pulled directly from the Wavefront Data Acquisition System (DAQ) after each exposure. The camera team is responsible for the DAQ and will provide its Application Programming Interface (API) and compilable client source code on a Linux-based computer, along with connectivity instructions. This differs from the nominal science operation mode, for which the images coming from the camera are directly sent to the data management team for processing and archiving at the National Center for Supercomputing Applications (NCSA).

Each detector of a corner raft sees a different field of the sky; therefore the stars on each the 2 detectors in each corner are different. In addition, compared to normal active or adaptive optics systems, the wavefront sensors have a large FOV, with numerous stars. In addition, the detectors are located 1.7 degrees off-axis, introducing vignetting and image distortion. For these reasons, image processing before using any curvature sensing algorithm is required to make the images usable. This includes basic image processing such as Instrumentation Signature Removal (ISR), ${ }^{16,17}$ source selection using a bright star catalog, source processing (deblending and intensity scaling) and master creation. Where applicable, the WEP utilizes common methods available in the LSST software stack. $^{16,17}$ Figure 3 shows the overall architecture of the WEP.

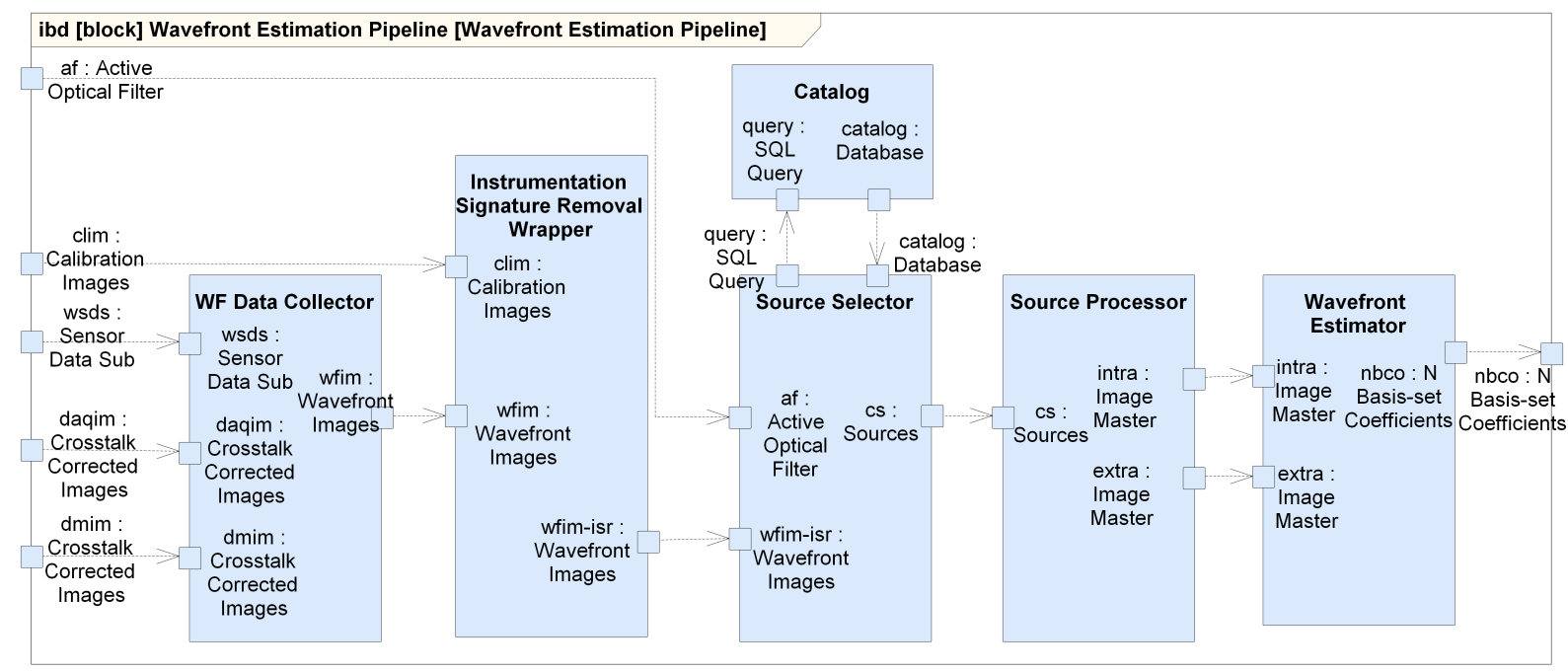

Figure 3. This diagram shows the different steps included in the WEP algorithm. These steps are described in the text.

\subsection{Preprocessing}

Wavefront Data Collector: During normal operation (see section 2.1) the wavefront sensor data is directly sent to the AOS from the camera subsystem using the DAQ through a push/pull interface. For the full array use case, the baseline is for the wavefront sensor data to go directly to the archive before being pulled by the AOCS. In this mode, the camera control system publishes an event, triggering the active optics system to pull the wavefront images from the 4 wavefront sensor detectors. For both cases, the images are cross-talk corrected. 
Instrument Signature Removal Wrapper: The WEP is compatible with the Instrument Signature Removal (ISR) developed by the DM team and will use some of its functions. The stack features used by the WEP are simple dark subtraction, flat field correction, gain and biases correction. The ISR will also include world coordinate system (WCS) correction. The other corrections required by the WEP are not part of the ISR and are described in the following paragraphs.

The Bright Star Catalog and Source Selector: Due to the large FOV of the wavefront sensor detectors, the source finding will rely on a bright star catalog. The impact of galaxies is expected to be minimal. The catalog contains the RA and DEC of bright stars only. It includes the star's limiting magnitude, and the saturation as a function of the LSST filter, and information about the number of neighbors falling in a predefined nearby surrounding region. The final acceptable number of neighbors within a certain region of interest and the size of that region of interest are currently being defined. The first instance of the catalog is expected to utilize the catalog developed by USNO; ${ }^{11}$ this catalog will be refined using LSST data during the lifetime of the survey. Figure 4 shows the pre-requisite and components of the source selector step.

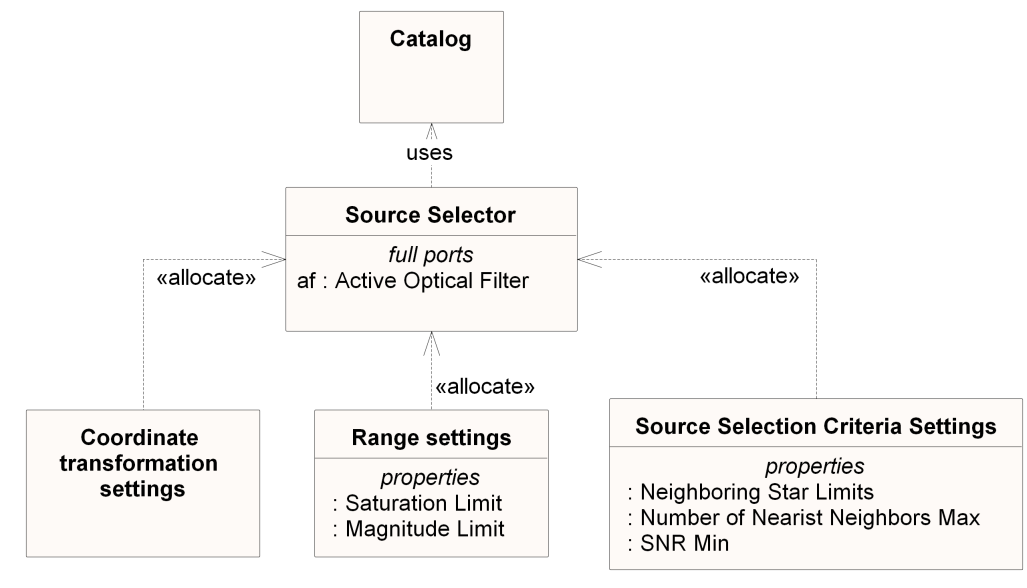

Figure 4. This diagram shows details of the source selection process, including items needed such as a bright star catalog or components of this step.

Creation of intra/extra focal plan image masters: The result of the source finding step is a set of postage stamps for each of the 8 detectors, including neighbors. The WEP then cleans the postage stamp images creating sub-images of donuts without any contamination from nearby stars. This process is called deblending. Because of the different magnitudes on each detector, the intensity from each sub-image is scaled to a same magnitude. Finally, all the images are summed to create a single pair of intra- and extra-focal images per sensor reference to as the master. These intra and extra masters can be seen as the input to regular curvature sensing.

The limiting magnitude will vary slightly depending on the condition of the night (seeing, cloud coverage, etc.). Therefore, the quality control is required to determine if a particular donut has the required signal to noise ratio $(\sim 10)$ to be used in the WEP without introducing extra residual errors.

\subsection{Wavefront Estimator}

Each set of intra and extra masters are then sent to the wavefront estimator. The wavefront sensor algorithm used for LSST is curvature sensing and has been described in detail in Xin et al. ${ }^{18}$ Due to the location of the wavefront sensors in the focal plane $(\sim 1.7$ degrees off axis) there is a need for distortion and vignetting corrections, preventing analytical mapping from the telescope aperture to the defocused image. In addition, since LSST has a large central obstruction (60\%) and fast beam (f-number of 1.23), the WEP uses a numerical solution, representing the mapping between the two sets of coordinates with $2 \mathrm{D}$ 10th-order polynomials. The 
off-axis distortion and the vignetting information comes from the optical design (Zemax and pupil information) and depend on the star location on the detector.

To determine a final algorithm, our strategy is to choose two well-established algorithms that are known to work for large f-number, on-axis systems, implement them as our baseline, then extend them to work with small f-number and off-axis sensors. As our two baseline algorithms we have chosen the iterative fast Fourier transform (FFT) method by Roddier and Roddier, ${ }^{19}$ and the series expansion technique by Gureyev and Nugent. ${ }^{20}$ More details are given in Xin et al. ${ }^{18}$

\subsection{Required Inputs and output}

Figure 5 provides further details on the inputs and sub steps that happen in the wavefront estimator box shown in Figure 3.

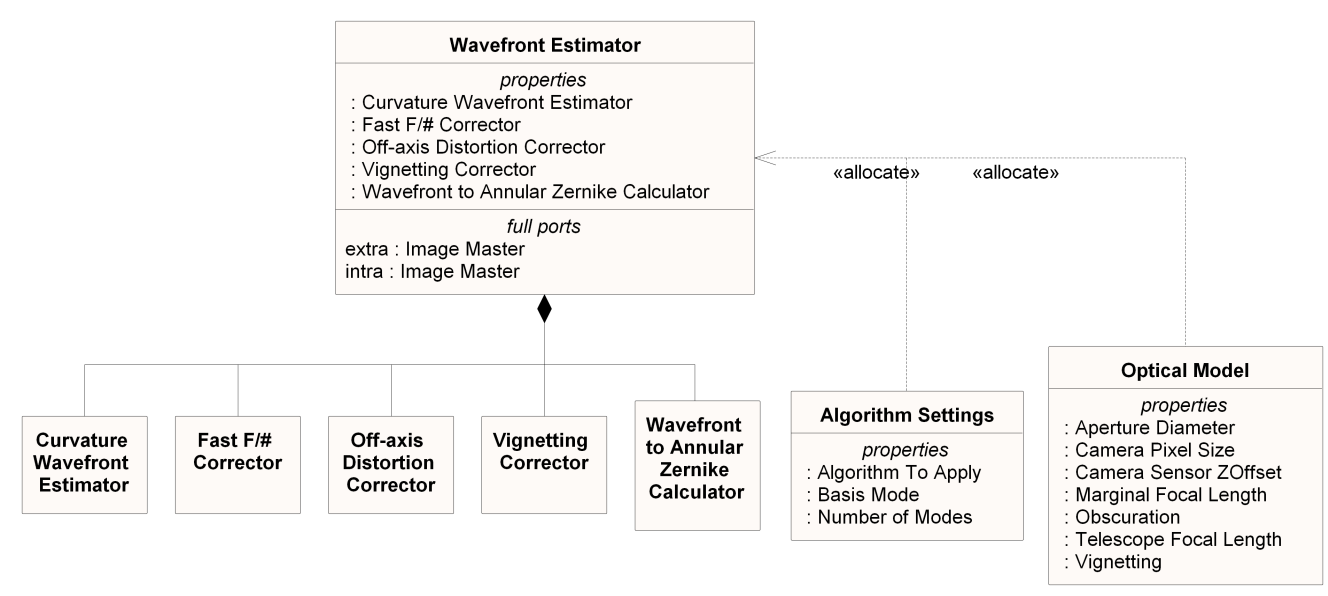

Figure 5. This diagram shows the details of the inputs of the different steps included in the wavefront estimator algorithm.

The output of the WEP is an annular Zernike decomposition of the wavefront. The output is communicated via Data Distribution System (DDS) ${ }^{14}$ or middleware to the active optics control system as a structure with three main elements for each sensor: the basis-set name, the number of terms, and the different coefficients. In addition, there is an image quality metric associated with each result.

\subsection{Pipeline Implementation}

WEP implementation will utilize Luigi, a platform independent Python package designed to simplify processing pipelines. It contains boilerplate code and the dependencies are decentralized. This removes the need for large configuration files since each task specifies its own dependencies. The use of Luigi will be limited to defining the pipeline task hierarchy. The processing of each task will be defined within its own package. This separates the responsibility for controlling execution order from the actual processing. The result is a set of Python classes with very simple implementations that clearly describe each task's dependencies, parameters, outputs, and responsibilities.

\section{ACTIVE OPTICS CONTROL SYSTEM}

This subsection describes the Active Optics Control System (AOCS). As mentioned above, the AOCS uses the wavefront measurements pushed by the WEP through the middleware to calculate the correction to send to the different control elements. The overall feedback algorithm can be separated into two steps: the optical state reconstructor and the control algorithm (called setpoint mapper in the block diagram presented in Figure 6). The degrees of freedom that the telescope possesses are the following:

- M1M3 shape, up to 20 bending modes 
- M2 shape, up to 20 bending modes

- M2 piston, $\mathrm{x} / \mathrm{y}$ decenter and $\mathrm{x} / \mathrm{y}$ tilt using the M2 hexapod ${ }^{7}$

- Camera piston, $\mathrm{x} / \mathrm{y}$ decenter and $\mathrm{x} / \mathrm{y}$ tilt using the camera hexapod/rotator ${ }^{7}$

The corrections, defined as bending modes and positions, are sent to the different correction elements controllers, namely, the M1M3 controller, M2 controller, M2 hexapod and camera hexapod, as shown on the right hand of Figure 6 . The number of bending modes is the optimum number to achieve the required image quality given by complete simulations. ${ }^{8}$ The output of the AOCS (and thus the AOS) is defined in terms of bending mode offsets for the M1M3 and M2 shapes as well as the hexapod setpoints. The offsets are relative to the LUT (or open model) defined in the introduction. The AOS sends setpoints to the component controllers responsible for moving the relevant axes. These controllers utilize open-loop models (possibly LUTs), influence matrices to convert from bending modes to forces, and custom electronics boards known as Inner Loop Controllers. ${ }^{4}$

Similarly to the wavefront estimation pipeline, the optical feedback control algorithm design is described in detail in a previous paper. ${ }^{8}$ The focus of this paper is on the architecture.

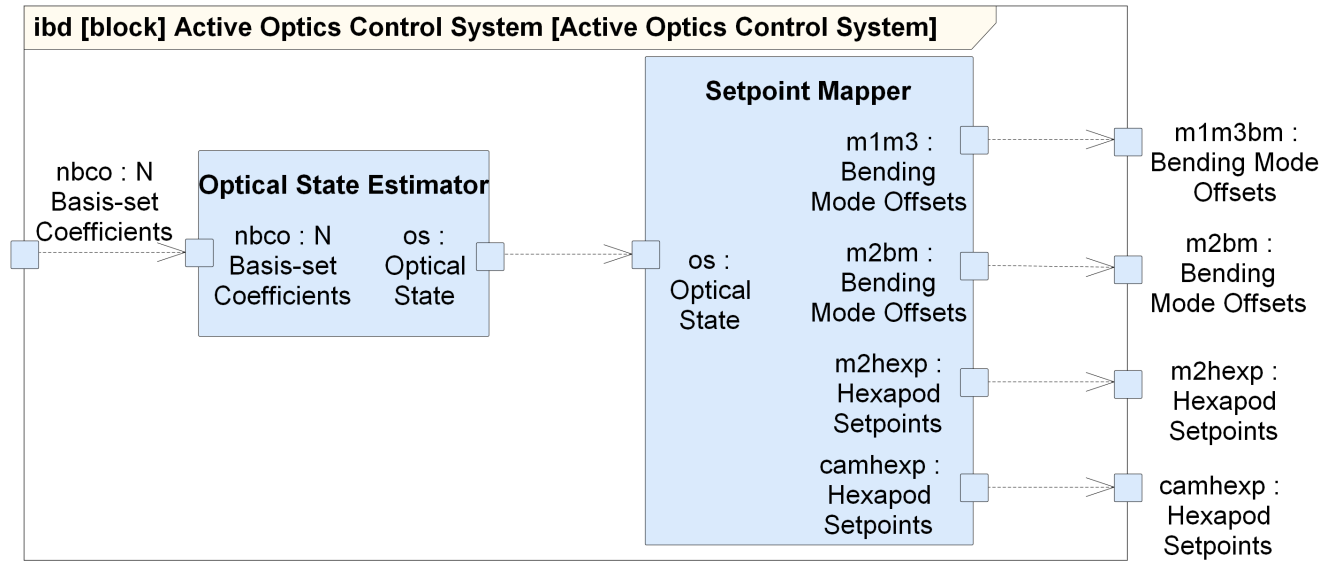

Figure 6. The block diagram shows the different steps needed to run the AOCS algorithm. This flow-down comes after the WEP flow-down shown in Figure 3. In this block diagram, the "setpoint mapper" is the control algorithm used to create the correction to improve the image quality. What is not shown in this graph is the sensitivity matrix needed by both the optical state estimator and the control algorithm.

Optical State Estimation: The AOCS derives the appropriate state of the optical system from the wavefront sensors in the presence of algorithmic, WCS and atmospheric noise errors. ${ }^{8}$ The state estimation is either done from the 4 sets of basis coefficients for normal operation or 189 sets for full array use case. The most conceptually simple version of this estimator uses the pseudo-inverse of the sensitivity matrix described at the end of this section.

Control algorithm (or setpoint mapper): Once the AOCS estimates the optical state of the telescope, it uses an optimal control determining using a cost function to derive the realistic bending modes to apply to M1M3 and M2 control systems as well as the M2 and camera hexapods. The result of this cost function provides the best optical quality with the smallest actuator action. It also sets the control authority between actuator groups (mirror bending modes and hexapod displacements). The cost function is built to allow the algorithm to calculate the solution that will optimize the image quality, such as by minimizing the variance of the Full Width Half Max (FWHM) over the large FOV. Furthermore, the cost function helps limit large actuator swings to avoid damage to the glass, as well as ensure smooth transitions between iterations. The FOV mapping uses the Gaussian Quadratic method. The temporal (sampling) behavior of the described controller is equivalent to 
an integral controller. Performance can possibly be further optimized by a more complex dynamic controller utilizing earlier system state estimates.

Sensitivity Matrix: The AOCS uses a sensitivity matrix calculated prior to any AOCS reconstruction. The sensitivity matrix describes the optical response of the system to the various controlled degrees of freedom. The columns of the sensitivity matrix correspond to the controlled degrees of freedom (described above). The rows of the sensitivity matrix correspond to the Zernike coefficients of the wavefront at the $\mathrm{N}$ sensor locations. This matrix A is used in both the state estimation process and the control algorithm. This sensitivity matrix is planned to be measured on-sky during commissioning. Note that the bending modes and Zernike coefficients are measured relative to a reference state. The sensitivity matrix is calculated offline after gathering on-sky data. It is not expected to change often and will not be modified during a night.

\section{CONCLUSIONS}

LSST is equipped with an active optics to optimize the image quality of the system. The active optics is a curvature sensing systemoptimized for the LSST wide field-of-view and optical design. The software architecture of the AOS is decomposed into two main components: the wavefront estimation pipeline (WEP) which estimates the residual wavefront from the open-loop model, and the active optics control system (AOCS) which calculates the M1M3 and M2 shapes as well as the M2 and camera hexapods positions. Both WEP and AOCS are complex entities that require severals inputs from other LSST subsystems and comprised of several steps. The AOS is right now in full development with a completion date in accordance with the Telescope and Site Integration Plan, late 2018 .

\section{ACKNOWLEDGMENTS}

This material is based upon work supported in part by the National Science Foundation through Cooperative Agreement 1258333 managed by the Association of Universities for Research in Astronomy (AURA), and the Department of Energy under Contract No. DE-AC02-76SF00515 with the SLAC National Accelerator Laboratory. Additional LSST funding comes from private donations, grants to universities, and in-kind support from LSSTC Institutional Members.

\section{REFERENCES}

[1] Kahn, S., "LSST final design overview," Proc. SPIE 9906, Paper 17, in press (2016).

[2] Gressler, W. et al., "LSST telescope and site status," Proc. SPIE 9906, Paper 19, in press (2016).

[3] Araujo, C., Jacques, S., Ming, L., Neill, D., Thomas, Sandrine, J., Vucina, T., and Gressler, W., "Overview of the LSST mirror system," Proc. SPIE 9906, Paper 20, in press (2016).

[4] Neill, Douglas, R., Muller, G., Hileman, E., Joe, D., Araujo, C., Gressler, W., Lotz, P., Dave, M., Sebag, J., Thomas, S., Warner, M., and Wiecha, O., "Final design of the LSST primary/tertiary mirror cell assembly," Proc. SPIE 9906, Paper 25, in press (2016).

[5] Kurita, N. et al., "Large Synoptic Survey Telescope camera design and construction," Proc. SPIE 9912, Paper 27, in press (2016).

[6] Neill, D., Angeli, G., Claver, C., Hileman, E., DeVries, J., Sebag, J., and Xin, B., "Overview of the LSST active optics system," Proc. SPIE 9150, 91500G-91500G-16 (2014).

[7] Sneed, R., Neill, D., Kidney, S., Araujo, C., Gressler, W., Lotz, P., Dave, M., Sebag, J., Sebring, T., A., Warner, M., and Wiecha, O., "Final design of the LSST hexapod and rotator," Proc. SPIE 9906, Paper 18 , in press (2016).

[8] Angeli, G. Z., Xin, B., Claver, C., MacMartin, D., Neill, D., Britton, M., Sebag, J., and Chandrasekharan, S., "Real time wavefront control system for the Large Synoptic Survey Telescope (LSST)," Proc. SPIE 9150, 91500H-91500H-16 (2014).

[9] Angeli, George, Z., Xin, B., Claver, C., Cho, M., Dribusch, C., Neill, D., Peterson, J., Sebag, J., and Thomas, S., "An integrated modeling framework for the Large Synoptic Survey Telescope (LSST)," Proc. SPIE 9911, Paper 46, in press (2016). 
[10] Peterson, J. R., Jernigan, J. G., Kahn, S. M., Rasmussen, A. P., Peng, E., Ahmad, Z., Bankert, J., Chang, C., Claver, C., Gilmore, D. K., Grace, E., Hannel, M., Hodge, M., Lorenz, S., Lupu, A., Meert, A., Nagarajan, S., Todd, N., Winans, A., and Young, M., "Simulation of astronomical images from optical survey telescopes using a comprehensive photon monte carlo approach," The Astrophysical Journal Supplement Series 218(1), 14 (2015).

[11] Monet, D. G., Levine, S. E., Canzian, B., Ables, H. D., Bird, A. R., Dahn, C. C., Guetter, H. H., Harris, H. C., Henden, A. A., Leggett, S. K., Levison, H. F., Luginbuhl, C. B., Martini, J., Monet, A. K. B., Munn, J. A., Pier, J. R., Rhodes, A. R., Riepe, B., Sell, S., Stone, R. C., Vrba, F. J., Walker, R. L., Westerhout, G., Brucato, R. J., Reid, I. N., Schoening, W., Hartley, M., Read, M. A., and Tritton, S. B., "The USNO-B Catalog," The Astrophysical Journal 125, 984-993 (2003).

[12] Connolly, A. J., Angeli, G. Z., Chandrasekharan, S., Claver, C. F., Cook, K., Ivezic, Z., Jones, R. L., Krughoff, K. S., Peng, E.-H., Peterson, J., Petry, C., Rasmussen, A. P., Ridgway, S. T., Saha, A., Sembroski, G., vanderPlas, J., and Yoachim, P., "An end-to-end simulation framework for the Large Synoptic Survey Telescope," Proc. SPIE 9150, 915014 (2014).

[13] Lotz, P., Dubois-Felsmann, Gregory, P., Lim, K.-T., Johnson, T., Chandrasekharan, S., Mills, D., Daly, P., Schumacher, G., Delgado, F., Pietrowicz, S., Selvy, B., Sebag, J., Marshall, S., Sundararaman, H., Contaxis, C., Bovill, R., and Jeness, T., "LSST control software component design," Proc. SPIE 9913, Paper 9, in press (2016).

[14] Mills, D., Schumacher, G., and Lotz, P., "LSST communications middleware implementation," Proc. SPIE 9906, Paper 204, in press (2016).

[15] Daly, Phil, N., Schumacher, G., Delgado, F., and Mills, D., "LSST OCS plan and status," Proc. SPIE 9913, Paper 112, in press (2016).

[16] Jenness, T., Bosch, J., Owen, R., Parejko, J., Sick, J., Swinbank, J., de Val-Borr, M., Dubois-Felsmann, G., Lim, K.-T., Lupton, Robert, H., Schellart, P., Krughoff, Simon, K., and Tollerud, Erik, J., "Investigating interoperability of the LSST data management software stack with astropy," Proc. SPIE 9913, Paper 16, in press (2016).

[17] Jurić, M., Kantor, J., Lim, K.-T., Lupton, R. H., Dubois-Felsmann, G., Jenness, T., Axelrod, T. S., Aleksić, J., Allsman, R. A., AlSayyad, Y., Alt, J., Armstrong, R., Basney, J., Becker, A. C., Becla, J., Bickerton, S. J., Biswas, R., Bosch, J., Boutigny, D., Carrasco Kind, M., Ciardi, D. R., Connolly, A. J., Daniel, S. F., Daues, G. E., Economou, F., Chiang, H.-F., Fausti, A., Fisher-Levine, M., Freemon, D. M., Gee, P., Gris, P., Hernandez, F., Hoblitt, J., Ivezić, Ž., Jammes, F., Jevremović, D., Jones, R. L., Bryce Kalmbach, J., Kasliwal, V. P., Krughoff, K. S., Lang, D., Lurie, J., Lust, N. B., Mullally, F., MacArthur, L. A., Melchior, P., Moeyens, J., Nidever, D. L., Owen, Russell aNeillnd Parejko, J. K., Peterson, J. M., Petravick, D., Pietrowicz, S. R., Price, P. A., Reiss, D. J., Shaw, R. A., Sick, J., Slater, C. T., Strauss, M. A., Sullivan, I. S., Swinbank, J. D., Van Dyk, S., Vujčić, V., Withers, A., Yoachim, P., and LSST Project, f. t., "The LSST Data Management System," Astronomical Data Analysis Software and Systems XXV proc., arXiv:1512.07914 (2015).

[18] Xin, B., Claver, C., Liang, M., Chandrasekharan, S., Angeli, G., and Shipsey, I., "Curvature wavefront sensing for the Large Synoptic Survey Telescope," Applied Optics 54, 9045- (Oct. 2015).

[19] Roddier, C. and Roddier, F., "Wave-front reconstruction from defocused images and the testing of groundbased optical telescopes.," Journal of the Optical Society of America A 10, 2277-2287 (1993).

[20] Gureyev, T. E. and Nugent, K. A., "Phase retrieval with the transport-of-intensity equation. II. Orthogonal series solution for nonuniform illumination," Journal of the Optical Society of America A: Optics 13(8), 1670-1682 (1996). 